\title{
Drifter dispersion in the Adriatic Sea: Lagrangian data and chaotic model
}

\author{
G. Lacorata $^{1}$, E. Aurell ${ }^{2}$, and A. Vulpiani ${ }^{3}$ \\ ${ }^{1}$ Dipartimento di Fisica, Università dell'Aquila, Via Vetoio 1, I-67010 Coppito, L'Aquila, Italy, \\ Istituto di Fisica dell'Atmosfera, CNR, Via Fosso del Cavaliere, I-00133 Roma, Italy, \\ and Dipartimento di Fisica, Università di Roma "La Sapienza", Piazzale Aldo Moro 5, I-00185 Roma, Italy \\ ${ }^{2}$ Department of Mathematics, Stockholm University, S-10691 Stockholm, Sweden \\ ${ }^{3}$ Istituto Nazionale Fisica della Materia, Unità di Roma 1 \\ and Dipartimento di Fisica, Università di Roma "La Sapienza”, Piazzale Aldo Moro 5, I-00185 Roma, Italy
}

Received: 8 November 1999 - Revised: 14 September 2000 - Accepted: 14 September 2000

\begin{abstract}
We analyze characteristics of drifter trajectories from the Adriatic Sea with recently introduced nonlinear dynamics techniques. We discuss how in quasi-enclosed basins, relative dispersion as a function of time, a standard analysis tool in this context, may give a distorted picture of the dynamics. We further show that useful information may be obtained by using two related non-asymptotic indicators, the Finite-Scale Lyapunov Exponent (FSLE) and the Lagrangian Structure Function (LSF), which both describe intrinsic physical properties at a given scale. We introduce a simple chaotic model for drifter motion in this system, and show by comparison with the model that Lagrangian dispersion is mainly driven by advection at sub-basin scales until saturation sets in.
\end{abstract}

Key words. Oceanography: General (marginal and semiclosed seas) - Oceanography: Physical (turbulence, diffusion, and mixing processes; upper ocean processes)

\section{Introduction}

Understanding the mechanisms of transport and mixing processes is an important and challenging task which has wide relevance from a theoretical point of view, e.g. for the study of diffusion and chaos in geophysical systems in general or for validating simulation results from a general circulation model. It is also a necessary tool in the analysis of problems of general interest and social impact, such as the dispersion of nutrients or pollutants in sea water with consequent effects on marine life and on the environment (Adler et al., 1996).

Recently, a number of oceanographic programs have been devoted to the study of the surface circulation of the Adriatic Sea by the observation of Lagrangian drifters within the larger framework of drifter-related research in the whole Mediterranean Sea (Poulain, 1999). The Adriatic Sea is a quasienclosed basin, about 800 long by $200 \mathrm{~km}$ wide, connected to

Correspondence to: G. Lacorata

(Guglielmo.Lacorata@aquila.infn.it) the rest of the Mediterranean Sea through the Otranto Strait. From a topographic point of view, three major regions can be considered. The northern part is the shallowest, about $100 \mathrm{~m}$ maximum depth, and extends down to the latitude of Ancona. The central part extends down to about $260 \mathrm{~m}$ in the Jabuka Pit, and the southern part extends from the Gargano promontory to the Otranto Strait. The southern part is the deepest, reaching about $1200 \mathrm{~m}$ in the South Adriatic Pit. Reviews on the oceanography of the Adriatic Sea can be found in Artegiani et al. (1997), Orlic et al. (1992), Poulain (1999) and Zore (1956).

Lagrangian data offer the opportunity to employ techniques of analysis well established in the theory of chaotic dynamical systems to study the behavior of actual trajectories and compare those with a kinematic model.

Let us assume that the Lagrangian drifters are passively advected in a two-dimensional flow, e.g. as would be the case in a frictionless barotropic approximation (Ottino, 1989; Crisanti et al., 1991):

$\frac{d x}{d t}=u(x, y, t)$ and $\frac{d y}{d t}=v(x, y, t)$,

where $(x(t), y(t))$ is the position of a fluid particle at time $t$ in terms of longitude and latitude, and $u$ and $v$ are the zonal and meridional velocity fields respectively.

For the Eulerian description of a geophysical system, one should in principle use numerical solutions of the NavierStokes equations (or other suitable equations, e.g. the quasigeostrophic model) to obtain the velocity fields. In practice, direct numerical simulation of these equations on oceanographic length scales is of course not possible, and one has to invoke approximations, i.e. turbulence modeling. This motivates one to use instead a simplified kinematic approach by adopting a given Eulerian velocity field. The criteria for the construction of such a field follows from phenomenological arguments and/or experimental observation and have recently been reviewed in Yang (1996) and Samelson (1996).

Let us consider the relationship between Eulerian and Lagrangian properties of a system. A wide range of literature on 
this topic (e.g. Ottino, 1989; Crisanti et al., 1991) allows us to state that, in general, Eulerian and Lagrangian behaviours are not strictly related to each other. It is not rare to have regular Eulerian behavior, e.g. a time-periodic velocity field co-existing with Lagrangian chaos or vice-versa.

In quasi-enclosed basins like the Adriatic Sea, a characterization of the mechanisms of the mixing is highly non-trivial. We first observe (see below for detailed discussion) that the use of the standard diffusion coefficients can have rather limitated applicability (see Artale et al., 1997). Classical studies on Lagrangian particles in ocean models already contain remarks on the intrinsic difficulties in using one-particle diffusion statistics (Taylor, 1921). In situations where the advective time is not much longer than the typical decorrelation time scale of the Lagrangian velocity, the diffusivity parameter related to small-scale turbulent motion cannot converge to its asymptotic value (Figueroa and Olson, 1994). On the other hand, a generalization of the standard Lyapunov exponent, the Finite-Scale Lyapunov Exponent (FSLE), originally introduced for the predictability problem (Aurell et al., 1996, 1997), has been shown to be a suitable tool to describe non-asymptotic properties of transport. This finitescale approach to Lagrangian transport measures effective rates of particle dispersion without assumptions about smallscale turbulent processes. For an alternative method, see Buffoni et al. (1997) and for a recent review and systematic discussion of non-asymptotic properties of transport and mixing in realistic cases, see Boffetta et al. (2000).

In this paper we report data analysis of surface drifter motion in the Adriatic Sea using relative dispersion, FSLE and Lagrangian Structure Function (LSF), a quantity related to the FSLE. We also introduce a chaotic model for the Lagrangian dynamics and use the FSLE and LSF characteristics to compare model and data. We show that it can be very difficult to obtain an estimate of the diffusion coefficient in a quasi-enclosed basin, and/or to look for deviations from the standard diffusion law. In fact, the time a cluster of particles takes to spread uniformly and reach the boundaries is not much longer than the largest characteristic time of the system. In contrast, the FSLE and the LSF do characterize the transport properties of Lagrangian trajectories at a fixed spatial scale. Finally, we will show that a simple kinematic model reproduces the data.

In Sect. 2 we describe the data set we have used as well as review relevant concepts and analysis techniques for Lagrangian transport and chaos. In Sect. 3 we introduce a kinematic model of the Lagrangian dynamics, and in Sect. 4, we compare the data and the model. Section 5 contains a summary and a discussion of the results.

\section{Data set and analysis techniques}

\subsection{Data set}

In a large drifter research program in the Mediterranean Sea, started in the late 80's and continued into the 90's, Lagrang- ian data from surface drifters deployed in the Adriatic sea have been recorded from December 1994 to March 1996. These drifters are similar to the CODE (COastal Dynamics Experiment) system (Davis, 1985) and they are designed to be sufficiently wind-resistant so as to effectively give a description of the circulation at their actual depth (1 meter). The drifters were tracked by the Argos Data Location and Collection System (DCLS) carried by the NOAA polarorbiting satellites. It is assumed that after data processing drifter positions are accurate to within $200-300 \mathrm{~m}$, and velocities to within $2-3 \mathrm{~cm} / \mathrm{s}$. For a description of the experimental program, see Poulain (1999) and for technical details about the treatment of raw data, see Hansen and Poulain (1996), Poulain et al. (1996) and Poulain and Zanasca (1998).

The data have been stored in separate files, one for each drifter. In the format used by us each file contains: a number of records (i.e. number of points of the trajectory); the time in days; the position of the drifter in longitude and latitude; the velocity of the drifter along the zonal and meridional directions; and the temperature in centigrade degrees. The sampling time is 6 hours. We can identify five main deployments on which we will concentrate our attention. Selecting the tracks by the time of the first record, it is easy to verify that these five subsets consist of drifters deployed in the same area in the Otranto strait near 19 degrees longitude east and 40 degrees latitude north. The experimental strategy of simultaneously releasing the drifters within a distance of some kilometers allows us to study dispersion quantitatively.

From a qualitative point of view, what we observe from the plot of all the trajectories (Fig. 1) is the shape of two (cyclonic) basin-wide gyres, located in the middle and southern regions respectively and, an anti-clockwise boundary current which moves the drifters north-westward along the east coast and south-eastward down the west coast. The latter is a permanent feature of the Adriatic sea (Poulain, 1999). On the other hand, it is known that within a year the pattern of basin-wide gyres may change between one, two and three gyres over a time-scale of months. The southern gyre is the most steady of the three. The data also suggests the presence of small scale structures, even though these are much more likely to be variable in time. The time-scale of the typical recirculation period around a basin-wide gyre is about one month and the time needed to travel along the coasts and complete one lap of the full basin is in the order of a few months.

\subsection{Analysis techniques}

We recall here some basic concepts about dynamical systems, diffusion and chaos, and the quantities that we shall use to characterize the properties of Lagrangian trajectories.

If we have $N_{c}$ clusters of initially close particles with each cluster containing $n_{k}$ elements, relative dispersion can be characterized by the diffusion coefficient

$D_{i}=\lim _{t \rightarrow \infty} \frac{1}{2 t} S_{i}^{2}(t)$ 


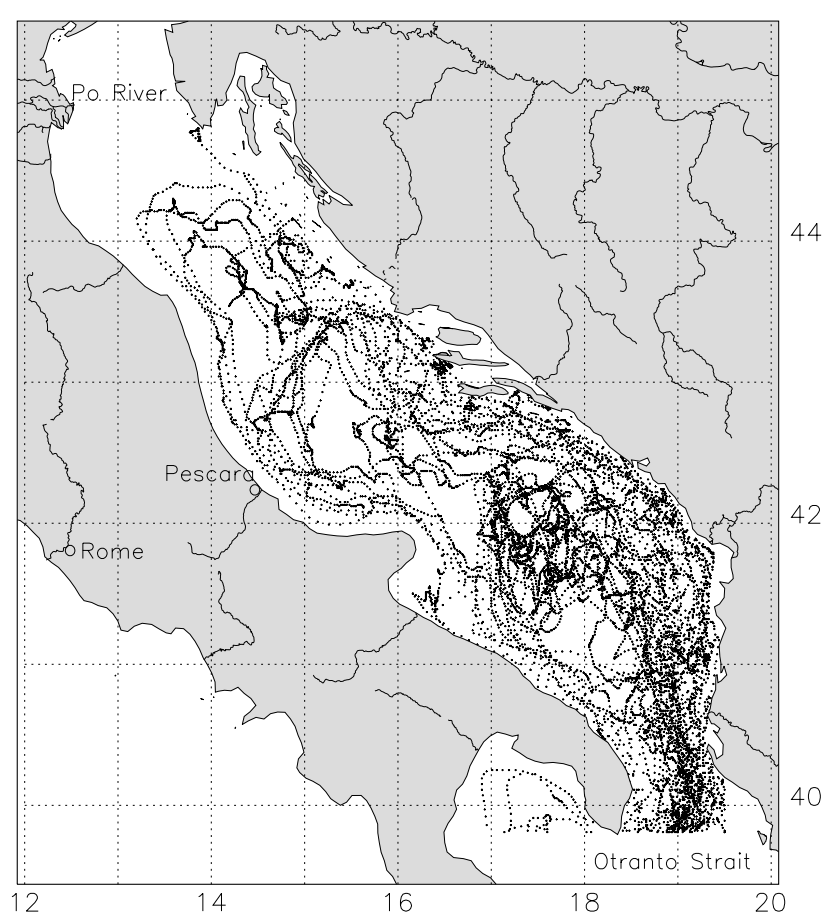

Fig. 1. Plot of the 37 drifter trajectories in the Adriatic Sea used for the data analysis. The longitude east and latitude north coordinates are in degrees. The drifters were deployed on the eastern side of the Otranto strait.

with

$S_{i}^{2}(t)=\frac{1}{N_{c}} \sum_{k=1}^{N_{c}} \frac{1}{n_{k}} \sum_{j=1}^{n_{k}}\left(x_{i}^{(k, j)}(t)-\left\langle x_{i}(t)\right\rangle^{(k)}\right)^{2}$

where

$\left\langle x_{i}(t)\right\rangle^{(k)}=\frac{1}{n_{k}} \sum_{j=1}^{n_{k}} x_{i}^{(k, j)}(t)$

$x_{i}^{(k, j)}$ is the $i$-th spatial coordinate of the $j$-th particle in the $k$-th cluster; $S^{2}=\sum_{i} S_{i}^{2}$ is the mean square displacement of the particles relative to their time evolving mean position. If $\delta(t)$ is the distance between two trajectories $\boldsymbol{x}^{(1)}$ and $\boldsymbol{x}^{(2)}$ in a cluster at time $t$, relative dispersion is defined as

$\left\langle\delta^{2}(t)\right\rangle=\left\langle\left\|\boldsymbol{x}^{(1)}(t)-\boldsymbol{x}^{(2)}(t)\right\|^{2}\right\rangle$

where the average is over all pairs of trajectories in the cluster. In a standard diffusive regime, $\boldsymbol{x}^{(1)}(t)$ and $\boldsymbol{x}^{(2)}(t)$ become independent variables and, for $t \rightarrow \infty$, we have $\left\langle\delta^{2}(t)\right\rangle$ $=2 S^{2}(t)$. In the following, we shall consider the cluster mean square radius $S^{2}(t)$ as a measure of relative dispersion. Absolute dispersion, which is defined as the mean square displacement from an initial position, will not be taken into account in our analysis.

If, in the asymptotic limit, $S_{i}^{2}(t) \sim t^{2 \alpha}$ with $\alpha=1 / 2$, we have the linear law of standard diffusion for the mean square displacement and the $D_{i}$ 's are finite. If $\alpha \neq 1 / 2$, we have a so-called anomalous diffusion (Bouchaud and Georges, 1990).

The difficulty which often arises when measuring the exponent $\alpha$ is that, due to the finite size of the domain, dispersion cannot reach its true asymptotic behavior. In other words, diffusion may not be observable over sufficiently large scales, i.e. much larger than the largest Eulerian length scale. Therefore, we cannot have a robust estimate of the exponent of the asymptotic power law. Moreover, the relevance of asymptotic quantities, like the diffusion coefficients, is questionable in the study of realistic cases concerning the transport problem in finite-size systems (Artale et al., 1997).

The diffusion coefficients characterize long-time (largescale) dispersion properties. In contrast, at short times (small scales) the relative dispersion is related to the chaotic behavior of the Lagrangian trajectories.

A quantitative measure of instability for the time evolution of a dynamical system (Lichtenberg and Lieberman, 1992) is commonly given by the Maximum Lyapunov Exponent (MLE) $\lambda$, which gives the rate of exponential separation of two nearby trajectories

$\lambda=\lim _{t \rightarrow \infty} \lim _{\delta(0) \rightarrow 0} \frac{1}{t} \ln \frac{\delta(t)}{\delta(0)}$

where $\delta(t)=\left\|\boldsymbol{x}^{(1)}(t)-\boldsymbol{x}^{(2)}(t)\right\|$ is the distance between two trajectories at time $t$. When $\lambda>0$ the system is said to be chaotic. There exists a well-established algorithm to numerically compute the MLE introduced by Benettin et al. (1980).

A characteristic time, $T_{\lambda}$, associated to the MLE is the predictability time, defined as the minimum time after which the error on the state of the system becomes larger than a tolerance value $\Delta$, if the initial uncertainty is $\delta$ (Lichtenberg and Lieberman, 1992):

$T_{\lambda}=\frac{1}{\lambda} \ln \frac{\Delta}{\delta}$

Let us recall that $\lambda$ is a mathematically well-defined quantity which measures the growth of infinitesimal errors. In physical terms, at any time, $\delta$ has to be much less than the characteristic size of the smallest relevant length of the velocity field. For example, in 3D fully developed turbulence, $\delta$ has to be much smaller than the Kolmogorov length.

When the uncertainty reaches non-infinitesimal sizes, i.e. macroscopic scales, the perturbation $\delta$ is governed by the nonlinear terms and that renders its growth rate a scale-dependent index (Aurell et al., 1996, 1997; Artale et al., 1997). It is useful to introduce the Finite Scale Lyapunov Exponent (FSLE), $\lambda(\delta)$. Assuming $r>1$ is a fixed amplification ratio and $\left\langle\tau_{r}(\delta)\right\rangle$ the mean time that $\delta$ takes to grow to $r \cdot \delta$, we have:

$\lambda(\delta)=\frac{1}{\left\langle\tau_{r}(\delta)\right\rangle} \ln r$

The average $\langle\cdot\rangle$ is performed over all the trajectory pairs in a cluster. We note the following properties of the FSLE: 
a) in the limit of infinitesimal separation between trajectories, $\delta \rightarrow 0$, the FSLE tends to the maximum Lyapunov exponent (MLE);

b) in case of standard diffusion, $\left\langle\delta(t)^{2}\right\rangle \sim t$, we find that $\lambda(\delta) \sim \delta^{-2}$ and the proportionality constant is of the order of the diffusion coefficient;

c) any slope $>-2$ for $\lambda(\delta)$ vs $\delta$ indicates super-diffusive behavior, i.e. non-neglectable correlations persist at long times and advection is still relevant;

d) in particular, when $\lambda(\delta)=$ constant over a range of scales, we have exponential separation between trajectories at a constant rate within that range of scales (chaotic advection).

Another interesting quantity related to the FSLE is the Lagrangian Structure Function (LSF) $v(\delta)$, defined as

$\nu(\delta)=\left\langle\left\|\frac{d \boldsymbol{x}^{\prime}}{d t}-\frac{d \boldsymbol{x}}{d t}\right\|\right\rangle_{\delta}$

where the value of the velocity difference is taken at the times for which the distance between the trajectories enters the scale $\delta$ and the average is performed over a large number of realizations. The LSF, $v(\delta)$, is a measure of the velocity at which two trajectories depart from each other, as a function of scale. By dimensional arguments, we expect that the LSF is proportional to the scale of the separation and to the FSLE:

$v(\delta) \sim \delta \lambda(\delta)$

so that we should find similar behavior for $\lambda(\delta)$ and $v(\delta) / \delta$, if independently measured.

In order to study the transport properties of the drifter trajectories, we have focused our interest on the measurement of $S_{i}^{2}(t), \lambda(\delta)$ and $v(\delta)$.

With regards to the practical definitions of the FSLE and the LSF, we have chosen a range of scales $\delta=\left(\delta_{0}, \delta_{1}, \ldots, \delta_{n}\right)$ separated by a factor $r>1$ such that $\delta_{i+1}=r \cdot \delta_{i}$ for $i=$ $0, n-1$. The ratio $r$ is often referred to as the "doubling" factor even though it is not necessarily equal to 2 , e.g. in our case we fixed it at $\sqrt{2}$. The $r$ value has naturally an inferior bound because of the temporal finite resolution of the trajectories (i.e. it cannot be arbitrarily close to 1 ) and it must be not much larger than 1 , if we want to resolve scale separation in the system.

The smallest threshold $\delta_{0}$ is placed just above the initial mean separation between two drifters, $\sim 10 \mathrm{~km}$, and the largest one $\delta_{n}$ is naturally selected by the finite size of the domain, $\sim 500 \mathrm{~km}$.

Following the same procedure, it is straightforward to compute the LSF as the mean velocity difference between two trajectories at the moment in which the separation reaches a scale $\delta$ :

$v(\delta)=\left\langle\sqrt{\left(u_{1}-u_{2}\right)^{2}+\left(v_{1}-v_{2}\right)^{2}}\right\rangle_{\delta}$ where the average is performed over the number of all the pairs within a set of particles, at the time in which $\left\|\boldsymbol{x}^{\prime}-\boldsymbol{x}\right\|=$ $\delta$.

In Sect. 4 below we shall show the results of our data analysis and compare them with the simulations from our chaotic model for the Lagrangian dynamics of the Adriatic drifters.

\section{The chaotic model}

In phenomenological kinematic modeling of geophysical flows, two possible approaches can be considered: stochastic and chaotic. Both procedures generally involve a mean velocity field, which gives the motion over large scales, and a perturbation which describes the action of the small scales. The model is stochastic or chaotic if the perturbation is a random process or a deterministic time-dependent function, respectively. Examples on kinematic mechanisms proposed to model the mixing process can be found in Bower (1991), Samelson (1992), Bower and Lozier (1994), Cencini et al. (1999).

The choice of one or the other depends on what one is interested in and what experimental information is available. In our case, we have opted for a deterministic model since there are indications that, at the sea surface, the instabilities of the Eulerian structures are primarily due to air-sea interactions, which are nearly periodic perturbations.

We want to consider a simple model. So let us assume as main features of the surface circulation the following elements: an anti-clockwise coastal current; two large cyclonic gyres; and some natural irregularities in the Lagrangian motion induced by the small scale structures.

Let us notice that the actual drifters may leave the Adriatic sea through the Otranto Strait, but we model our domain with a closed basin in order to study the effects of the finite scales on the transport and treat it like a 2D system, since the drifters explore the circulation in the upper layer of the sea within the first meters of water.

On the basis of the previous considerations, we introduce our kinematic model for the Lagrangian dynamics. Under the incompressibility hypothesis we write a $2 \mathrm{D}$ velocity field in terms of a stream function:

$u=-\frac{\partial \Psi}{\partial y}$ and $v=\frac{\partial \Psi}{\partial x}$.

Let us write our stream function as a sum of three terms:

$\Psi(x, y, t)=\Psi_{0}(x, y)+\Psi_{1}(x, y, t)+\Psi_{2}(x, y, t)$

defined as follows:

$$
\begin{aligned}
\Psi_{0}(x, y)= & \frac{C_{0}}{k_{0}}\left[-\sin \left(k_{0}(y+\pi)\right)+\cos \left(k_{0}(x+2 \pi)\right)\right] \\
\Psi_{1}(x, y, t)= & \frac{C_{1}}{k_{1}} \sin \left(k_{1}\left(x+\epsilon_{1} \sin \left(\omega_{1} t\right)\right)\right) \\
& \cdot \sin \left(k_{1}\left(y+\epsilon_{1} \sin \left(\omega_{1} t+\phi_{1}\right)\right)\right)
\end{aligned}
$$



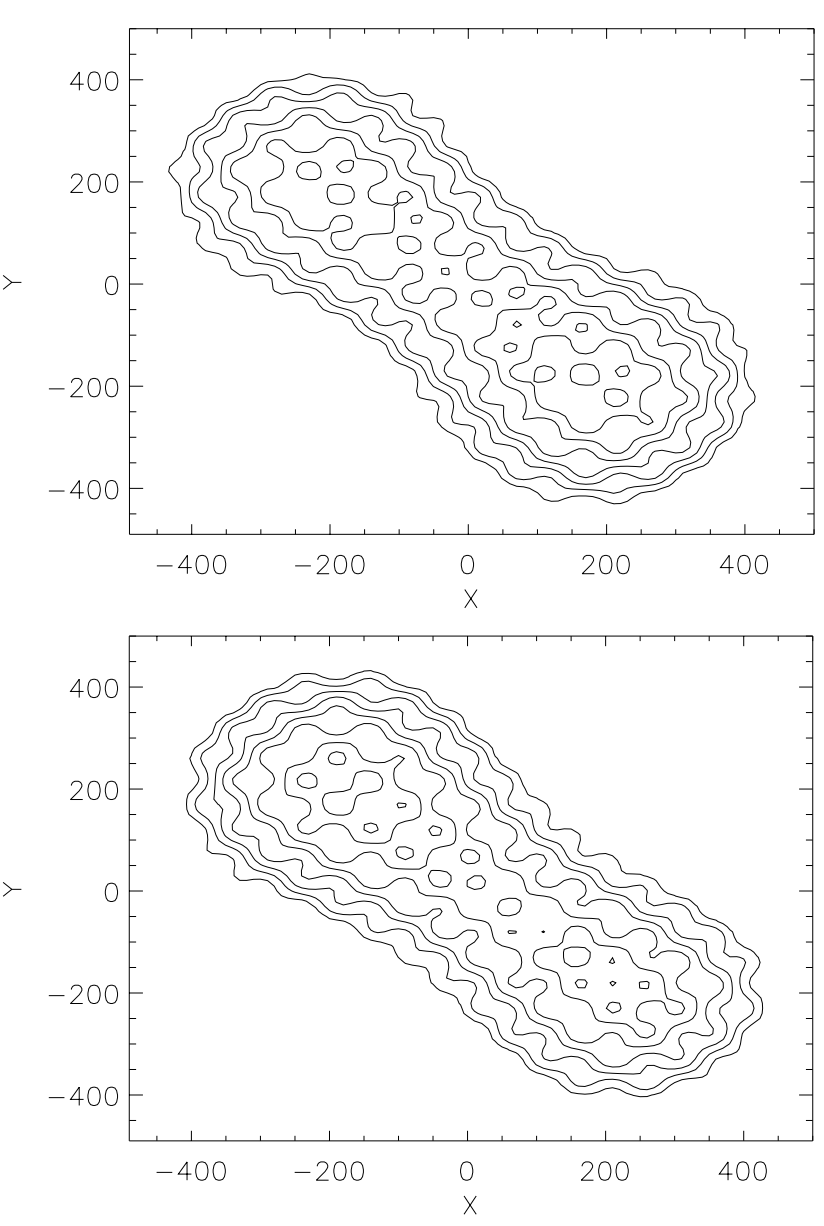

Fig. 2. Model stream function isolines at (a) $t=0$ and (b) $t=$ $T_{1} / 2$, with $T_{1} \sim 30$ days. The boundary of the domain is the zero isoline. The coordinates $(x, y)$ are in $\mathrm{km}$.

$$
\begin{aligned}
\Psi_{2}(x, y, t)= & \frac{C_{2}}{k_{2}} \sin \left(k_{2}\left(x+\epsilon_{2} \sin \left(\omega_{2} t\right)\right)\right) \\
& \cdot \sin \left(k_{2}\left(y+\epsilon_{2} \sin \left(\omega_{2} t+\phi_{2}\right)\right)\right)
\end{aligned}
$$

where $k_{i}=2 \pi / \lambda_{i}$, for $i=0,1,2$, the $\lambda_{i}$ are the wavelengths of the spatial structure of the flow; analogously $\omega_{j}=2 \pi / T_{j}$, for $j=1,2$, and the $T_{j}$ are the periods of the perturbations. In the non-dimensional expression of the equations, the units of length and time have been set to $200 \mathrm{~km}$ and 5 days, respectively. The choice of the values of the parameters is discussed below.

The stationary term $\Psi_{0}$ defines the boundary large scale circulation with positive vorticity. $\Psi_{1}$ contains the two cyclonic gyres and it is explicitly time-dependent through a periodic perturbation of the streamlines. The term $\Psi_{2}$ gives the motion over scales smaller than the size of the large gyres and it is time-dependent as well. A plot of the $\Psi$-isolines at fixed time is shown in Fig. 2. The actual basin is the inner region with negative $\Psi$ values and the zero isoline is taken as a dynamical barrier which defines the boundary of the domain.

The main difference with reality is that the model domain is strictly a closed basin, whereas the Adriatic Sea communicates with the rest of the Mediterranean through the Otranto
Strait. This is not crucial as long as we observe the two evolutions of experimental and model trajectories within time scales smaller than the mean exit time from the sea, typically of the order of a few months. Furthermore, the presence of the quasi-steady cyclonic coastal current is compatible with the interplay between the Po river southward inflow at the north-western side and the Otranto channel northward inflow at the south-eastern side of the sea.

The non-stationarity of the stream function is a necessary feature of a 2D velocity field in order to have Lagrangian chaos and mixing properties. That is, so that a fluid particle will visit any portion of the domain after a sufficiently long interval of time.

We have chosen the parameters as follows. The velocity scales $C_{0}, C_{1}$ and $C_{2}$ are all equal to 1 which, in physical dimensions, corresponds to $\sim 0.5 \mathrm{~m} / \mathrm{s}$. The wave numbers $k_{0}, k_{1}$ and $k_{2}$ are fixed at $1 / 2,1$ and $4 \pi$, respectively. In Fig. $2 \mathrm{a}, \mathrm{b}$ we can see two snapshots of the streamlines at fixed time. The length scales of the model Eulerian structures are of $\sim 1000 \mathrm{~km}$ (coastal current), $\sim 200 \mathrm{~km}$ (gyres) and $\sim 50$ $\mathrm{km}$ (eddies). The typical recirculation times for gyres and eddies turns out to be of the order of 1 month and a few days, respectively.

With regards to the time-dependent terms in the stream function, the pulsations are $\omega_{1}=1$ and $\omega_{2}=2 \pi$, which determine oscillations of the two large-scale vortices over a period $T_{1} \simeq 30$ days and oscillations of the small-scale vortices over a period $T_{2} \simeq 5$ days. The respective oscillation amplitudes are $\epsilon_{1}=\pi / 5$ and $\epsilon_{2}=\epsilon_{1} / 10$ which correspond to $\sim 100 \mathrm{~km}$ and $\sim 10 \mathrm{~km}$.

The choice of the phase factors $\phi_{1}$ and $\phi_{2}$ determines how much the vortex pattern changes during a perturbation period. We have chosen to set both $\phi_{1}$ and $\phi_{2}$ to $\pi / 4$. This choice of the parameters for the time-dependent terms in the stream function is only supposed to be physically reasonable, for the experimental data give us limited information about the time variability of the Eulerian structures.

The chaotic advection (Ottino, 1989; Crisanti et al., 1991), occurring in our model makes an ensemble of initially close trajectories spread apart from one another, until the size of the mean relative displacement reaches a saturation value corresponding to the finite length scale of the domain.

The scale-dependent degree of chaos is given by the FSLE. Due to the relatively sharp separation between large and small scales in the model, we expect $\lambda(\delta)$ to display a steplike behavior with two plateaus, one for each characteristic time, and a cut-off at scales comparable with the size of the domain. In the limit of small perturbations, the FSLE gives an estimate of the MLE of the system.

The LSF $v(\delta)$, on the other hand, is expected to be proportional to the size of the perturbation and to $\lambda$, as discussed in the introduction. Therefore, the quantity $v(\delta) / \delta$ is expected to be qualitatively proportional to $\lambda(\delta)$, in the sense that the mean slopes have to be compatible with each other.

In the following section we will show results of our simulations together with the outcome of the data analysis. 


\section{Comparison between data and model}

The statistical quantities relative to the drifter trajectories have been computed according to the following prescription. The number of selected drifters for the analysis is 37 , distributed in 5 different deployments in the Strait of Otranto, containing, respectively, 4, 9, 7, 7 and 10 drifters. These are the only drifter trajectories out of the whole data set which are long enough to study the Lagrangian motion on the basin scale. To obtain the highest statistics possible, at the price of losing information on the seasonal variability, the times of all of the 37 drifters are measured as $t-t_{0}$, where $t_{0}$ is the time of deployment. Moreover, to restrict the analysis only to the Adriatic basin, we impose the condition that a drifter is discarded as soon as its latitude moves south of $39.5 \mathrm{~N}$ or its longitude exceeds 19.5 E. Let us consider the reference frame in which the axes are aligned, respectively, with the short side, orthogonal to the coasts, which we call the transverse direction and the long side, along the coasts, which we call the longitudinal direction.

Before the presentation of the data analysis, let us briefly discuss the problem of finding characteristic Lagrangian times. A first obvious candidate is

$\tau_{L}^{(1)}=\frac{1}{\lambda}$

Of course $\tau_{L}^{(1)}$ is related to small scale properties. Another characteristic time, at least if the diffusion is standard, is the so-called integral time scale (Taylor, 1921)

$\tau_{L}^{(2)}=\frac{1}{\left\langle v^{2}\right\rangle} \int_{0}^{\infty} C(\tau) d \tau$

where $C(\tau)=\sum_{i=1}^{d}\left\langle v_{i}(t) v_{i}(t+\tau)\right\rangle$ is the Lagrangian velocity correlation function and $\left\langle v^{2}\right\rangle$ is the velocity variance. We want to stress that it is always possible (at least in principle) to define $\tau_{L}^{(1)}$ while to compute $\tau_{L}^{(2)}$ (the integral time scale) it is necessary to be in a standard diffusion case (Taylor, 1921).

The relative dispersion curves along the two natural directions of the basin for data and model trajectories are shown in Figs. 3a and 3b. The curves from the numerical simulation of the model are computed observing the spreading of a cluster of $10^{4}$ initial conditions. When a particle reaches the boundary $(\Psi=0)$, it is eliminated. Along with observational and simulation data, we also plot a straight line corresponding to a standard diffusion with coefficient $10^{3} \mathrm{~m}^{2} / \mathrm{s}$, (Falco et al., 2000). We discuss this comparison below. Considering the effective diffusion properties, one should expect that the shape of $S_{i}^{2}(t)$, before the saturation regime, can still be affected by the action of the coherent structures. Actually, neither the data nor the model dispersion curves display a clear power-law behavior, and are indeed quite irregular. The growth of the mean square radius of a cluster of drifters appears still strongly affected from the details of the system and the saturation begins no later than $\sim 1$ month $(\sim$ the largest characteristic Lagrangian time). This prevents any attempt at defining a diffusion coefficient for the effective dispersion
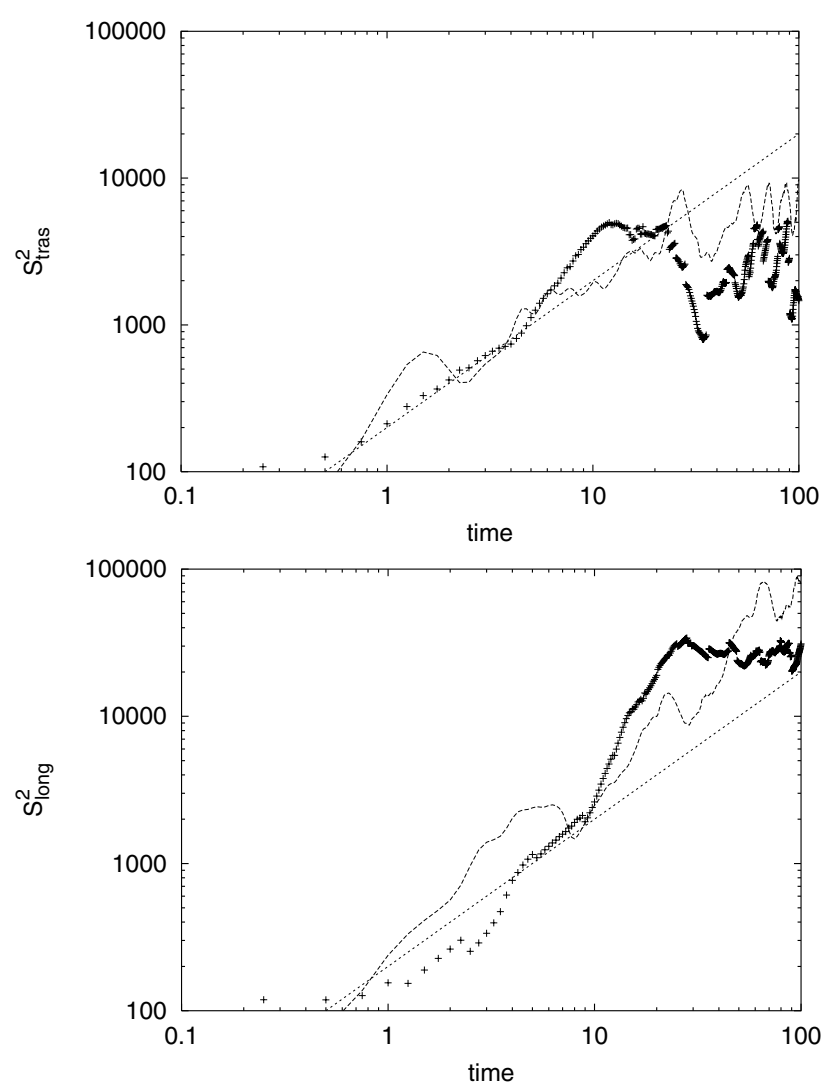

Fig. 3. Relative dispersion curves for data (plus symbol) and model (dashed) trajectories along the two natural directions in the basin geometry: (a) transverse component, (b) longitudinal component. The time is measured in days and the dispersion in $\mathrm{km}^{2}$. The experimental curve is computed over the 37 drifter trajectories; the model curve is computed over a cluster of $10^{4}$ particles, initially placed at the border of the southern gyre with a mean square displacement of $\sim 50 \mathrm{~km}^{2}$. The straight line with slope 1 has been plotted for comparison with a standard diffusive scaling with a corresponding diffusion coefficient $\sim 10^{3} \mathrm{~m}^{2} / \mathrm{s}$, typical of marine turbulent motions.

in this system. Although the saturation values are very similar, we can see that, in the intermediate range, the agreement between observation and simulation is not good. We point out that the trouble in reproducing the drifter dispersion in time does not depend much on the statistics. It makes no difference whether there are 37 (data) or $10^{4}$ (model) trajectories. The problem is that the classic relative dispersion is not the most suitable quantity to be measured (irregular behavior even at high statistics).

Let us now discuss the FSLE results. The curve measured from the data has been averaged over the total number of pairs out of 37 trajectories $(\sim 700)$, under the condition that the evolution of the distance between two drifters is no longer followed when any of the two exits the Adriatic basin (see above). In Fig. 4 the FSLE's for data and model are plotted. Phenomenologically, fluid particle motion is expected to be faster at small scales and slower at large scales. The decrease of $\lambda(\delta)$ at increasing $\delta$ reflects the presence of several scales 


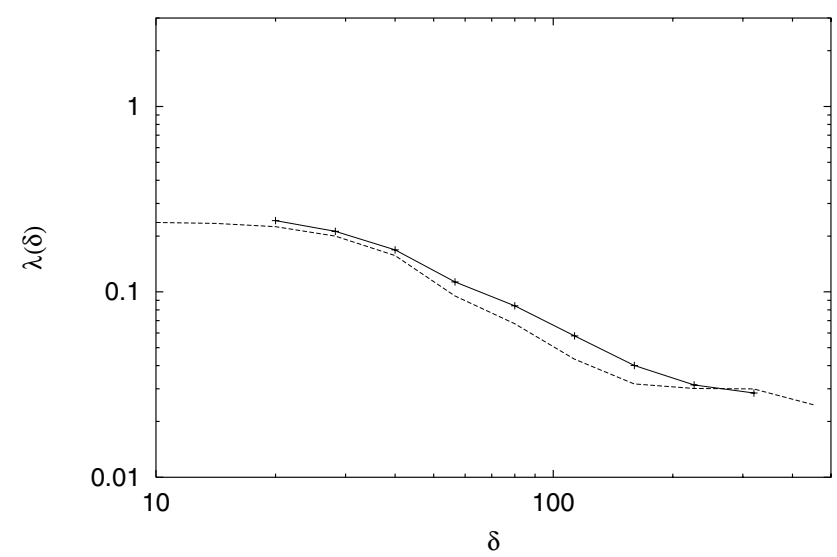

Fig. 4. Finite-Scale Lyapunov Exponent for data (continuous line) and model (dashed line) trajectories. $\delta$ is in $\mathrm{km}$ and $\lambda(\delta)$ is in day ${ }^{-1}$. The experimental FSLE is computed over all the pairs of trajectories out of 37 drifters; the FSLE from the model is averaged over $10^{4}$ simulations. The simulated $\lambda(\delta)$ has a step-like behavior with one plateau at small scales $(<50 \mathrm{~km})$ and one at basin scales $(>100$ $\mathrm{km}$ ), corresponding to doubling times of $\sim 3$ days and $\sim 30$ days, respectively.

of motions (at least two) involved in the dynamics. In particular, looking at the values of $\lambda(\delta)^{-1}$ at the extreme points of the $\delta$-range, we see that small-scale (mesoscale) dispersion has a characteristic time $\sim 4$ days and the large-scale (gyre scale) dispersion has a characteristic time $\sim 1$ month. The ratio between gyre scale and mesoscale is of the same order as the ratio between the inverse of their respective characteristic times $(\sim 10)$, so the slope of $\lambda(\delta)$ at intermediate scales is about -1 . The fact that the slope is larger than -2 indicates that relative dispersion is faster than standard diffusion up to sub-basin scales, i.e. Lagrangian correlations are non-vanishing due to coherent structures. It is interesting to compare this Lagrangian technique of measuring the effective Lagrangian dispersion on finite scales to the more traditional technique of extracting a (standard) diffusivity parameter from the reconstruction of the small-scale anomalies in the velocity field (Falco et al., 2000). Estimates of the zonal and meridional diffusivity in Falco et al. (2000) are of the order of $\sim 10^{3} \mathrm{~m}^{2} / \mathrm{s}$ and are compatible with the value of the effective finite-scale diffusive coefficient given by the FSLE, defined as $\lambda(\delta) \cdot \delta^{2}$, computed at $\delta=20 \mathrm{~km}(\sim$ the mesoscale).

The FSLE computed in the numerical simulations shows two plateaus, one at small scales and the other at large scales, describing a system with two characteristic time scales and presents the same behavior, both qualitative and quantitative, as the FSLE is computed for the drifter trajectories.

It is worth noting that it is much simpler for the model to reproduce, even quantitatively, the relation between characteristic times and scales of the drifter dynamics (FSLE) rather than the behavior of the relative dispersion in time.

The LSV in Fig. 5 shows that the behavior of $v(\delta)$, the mean velocity difference between two particle trajectories as

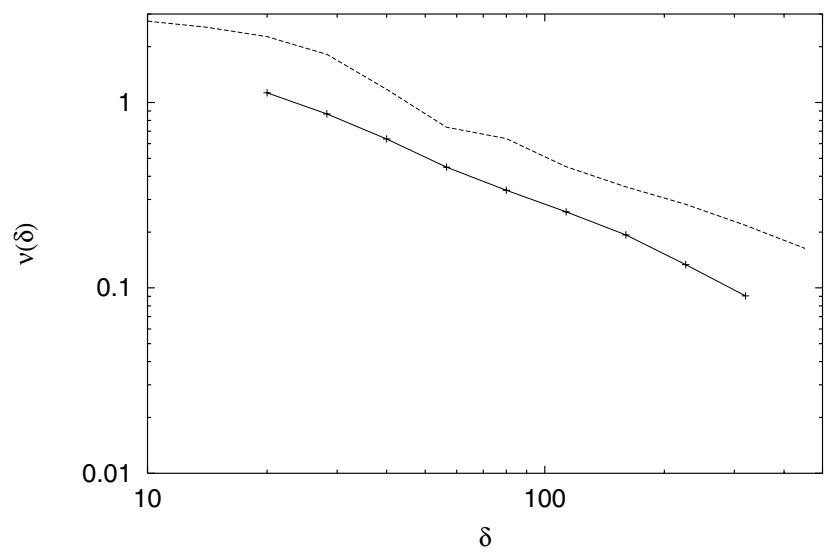

Fig. 5. Lagrangian Structure Function for data (continuous line) and model (dashed line) trajectories. $\delta$ is in $\mathrm{km}$ and $v(\delta) / \delta$ is in day ${ }^{-1}$. The experimental LSF is computed over all the pairs of drifters; the LSF from the model is averaged over $10^{4}$ simulations.

the scale of the separation varies, is compatible with the behavior of the FSLE as expected by dimensional arguments, i.e. $v(\delta) / \delta$, the LSF divided by the scale at which it is computed, has the same slope as $\lambda(\delta)$. This accounts for the robustness of the information given by the finite-scale analysis.

We see the theoretical predictions of FSLE and LSV are fairly well comparable with the corresponding quantities observed from the data, if we consider the relatively simple model which we used for the numerical simulations. Of course, an agreement exists due to the appropriate choice of the parameters of the model, capable of reproducing the correct relation between scales of motion and characteristic times, and because large-scale ( $\sim$ sub-basin scales) Lagrangian dispersion is weakly dependent on the small-scale $(\sim$ mesoscale) details of the velocity field.

\section{Discussion and conclusions}

In this paper we have analyzed an experimental data set recorded from Lagrangian surface drifters deployed in the Adriatic sea. The data span the period from December 1994 to March 1996, during which five sets of drifters were released at different times in the vicinity of the same point on the eastern side of the Otranto Strait. Adopting a technique borrowed from the theory of dynamical systems, we studied the Lagrangian transport properties by measuring relative dispersions $S_{i}^{2}$, finite-scale Lyapunov exponents $\lambda(\delta)$, and Lagrangian structure function $v(\delta)$. Relative dispersion, as function of time, does not provide much information, except for an idea of the size of the domain where saturation sets in at long times. The behavior of $S_{i}^{2}$ looks quite irregular and this is due not to poor statistics but rather to intrinsic reasons. In contrast, the results obtained with the FSLE, i.e. dispersion rates at different scales of motion, give a more useful description of the properties of the drifter spreading. In particular, $\lambda(\delta)$ detects the characteristic times associated with 
the Eulerian characteristic lengths of the system.

We have also introduced a simple chaotic model of the Lagrangian evolution and compared it with the observations. In our point of view, the actual meaning of the chaotic model, in relation to the behavior of the drifters, is not the bestfitting model. We do not claim that the quite difficult task of modeling the marine surface circulation driven by wind forcing can be exploited by a simple dynamical system. But a simple dynamical system can give satisfactory results if we are interested in large-scale properties of Lagrangian dispersion, since they depend much on the topology of the velocity field and only slightly on the small-scale details of the velocity structures. In this respect, chaotic advection, very likely present in every geophysical fluid flow, is crucial for what concerns tracer dispersion, since it can easily overwhelm the effects of small-scale turbulent motions on large-scale transport (Crisanti et al., 1991). Even when a standard diffusivity parameter can be computed from the variance and the self-correlation time of the Lagrangian velocity, its relevance for reproducing the effective dispersion on finite scales, in presence of coherent structures, is questionable. In addition, practical difficulties arising from both finite resolution and boundary effects suggest a revision of the analysis techniques to be used for studying Lagrangian motion on finite scales, i.e. in non-asymptotic conditions. Considering that, generally, there is more physical information in a scale-dependent indicator $(\lambda(\delta))$ rather than in a time function $\left(S^{2}(t)\right)$, we come to the conclusion that the FSLE is a more appropriate tool of investigation of finite-scale transport properties. It is important to remark (Aurell et al, 1996; Artale et al., 1997; Boffetta et al., 2000) that, in realistic cases, $\lambda(\delta)$ is not just another way to look at $S^{2}(t) v s t$, in particular it is not true that $\lambda(\delta)$ behaves like $\left(d \ln S^{2}(t) / d t\right)_{S^{2}=\delta^{2}}$. This is because $\lambda(\delta)$ is a quantity which characterizes Lagrangian properties at the scale $\delta$ in a non ambiguous way. On the contrary, $S^{2}(t)$ can depend strongly on $S^{2}(0)$, so that, in non-asymptotic conditions, it is relatively easy to get erroneous conclusions only by looking at the shape of $S^{2}(t)$. In fact, at a given time, relative dispersion inside a sub-cluster of drifters can be rather different from other sub-clusters, e.g. due to fluctuations in the cross-over time between exponential and diffusive regimes. Therefore, when performing an average over the whole set of trajectories, one may obtain a quite spurious and inconclusive behavior. On the other hand, we have seen that the analysis of FSLE (and LSF) and the studying of the transport properties at a given spatial scale, rather than at a given time, can provide more reliable information on the relative dispersion of tracers.

Acknowledgements. The drifter data set used in this work was kindly made available to us by P.-M. Poulain. We warmly thank J. Nycander, P.-M. Poulain, R. Santoleri and E. Zambianchi for constructive readings of the manuscript and for clarifying discussions about oceanographic matters. We also thank E. Bohm, G. Boffetta, A. Celani, M. Cencini, K. Döös, D. Faggioli, D. Fanelli, S. Ghirlanda, D. Iudicone, A. Kozlov, E. Lindborg, S. Marullo, P. Muratore-Ginanneschi and V. Rupolo for useful discussions.

This work was supported by a European Science Foundation "TAO exchange grant" (G. L.), by the Swedish Natural Science Research Council under contract M-AA/FU/MA 01778-334 (E. A.) and the Swedish Technical Research Council under contract 97-855 (E. A.), and by the I.N.F.M. "Progetto di Ricerca Avanzata TURBO" (A.V.) and MURST, program 9702265437 (A. V.). G. L. thanks the K.T.H. (Royal Institute of Technology) in Stockholm for hospitality. We thank the European Science Foundation and the organizers of the 1999 Tao Study Center for invitations, and for an opportunity to write up this work.

\section{References}

Adler, R. J., Müller, P., and Rozovskii, B. (Eds.), Stochastic modeling in physical oceanography, Birkhäuser, Boston, 1996.

Artale, V., Boffetta, G., Celani, A. , Cencini, M., and Vulpiani, A., Dispersion of passive tracers in closed basins: beyond the diffusion coefficient. Phys. of Fluids, 9, 3162, 1997.

Artegiani, A., Bregant, D., Paschini, E., Pinardi, N., Raicich, F., and Russo, A., The Adriatic Sea general circulation, parts I and II. J. Phys. Oceanogr., 27, 8, 1492-1532, 1997.

Aurell, E., Boffetta, G., Crisanti, A., Paladin, G., and Vulpiani, A., Predictability in systems with many degrees of freedom, Physical Review E, 53, 2337, 1996.

Aurell, E., Boffetta, G., Crisanti, A., Paladin, G., and Vulpiani, A., Growth of non-infinitesimal perturbations in turbulence, Phys. Rev. Lett., 77, 1262-1265, 1996.

Aurell, E., Boffetta, G., Crisanti, A., Paladin, G., and Vulpiani, A., Predictability in the large: an extension of the concept of Lyapunov exponent, J. of Phys. A, 30, 1, 1997.

Benettin, G., Galgani, L., Giorgilli, A., and Strelcyn, J. M., Lyapunov characteristic exponents for smooth dynamical systems and for Hamiltonian systems: a method for computing all of them, Meccanica, 15, 9, 1980.

Boffetta, G., Celani, A., Cencini, M., Lacorata, G., and Vulpiani, A., Non-asymptotic properties of transport and mixing, Chaos, Vol. 10, 1, 50-60, 2000.

Boffetta, G., Cencini, M., Espa, S., and Querzoli, G., Experimental evidence of chaotic advection in a convective flow, Europhys. Lett. 48, 629-633, 1999.

Bouchaud, J. P. and Georges, A., Anomalous diffusion in disordered media: statistical mechanics, models and physical applications, Phys. Rep., 195, 127, 1990.

Bower, A. S., A simple kinematic mechanism for mixing fluid parcels across a meandering jet, J. Phys. Oceanogr., 21, 173, 1991.

Bower, A. S. and Lozier, M. S., A closer look at particle exchange in the Gulf Stream, J. Phys. Oceanogr., 24, 1399, 1994.

Buffoni, G., Falco, P., Griffa, A., and Zambianchi, E., Dispersion processes and residence times in a semi-enclosed basin with recirculating gyres. The case of Tirrenian Sea, J. Geophys. Res., 102, C8, 18699, 1997.

Cencini, M., Lacorata, G., Vulpiani, A., and Zambianchi, E., Mixing in a meandering jet: a Markovian approach, J. Phys. Oceanogr. 29, 2578-2594, 1999.

Crisanti, A., Falcioni, M., Paladin, G., and Vulpiani, A., Lagrangian Chaos: Transport, Mixing and Diffusion in Fluids, La Rivista del Nuovo Cimento, 14, 1, 1991.

Davis, E. E., Drifter observation of coastal currents during CODE. The method and descriptive view, J. Geophys. Res., 90, 47414755, 1985. 
Falco P., Griffa, A., Poulain, P.-M., and Zambianchi, E., Transport properties in the Adriatic Sea as deduced from drifter data, J. Phys. Oceanogr., in press, 2000.

Figueroa, H. O. and Olson, D. B., Eddy resolution versus eddy diffusion in a double gyre GCM. Part I: the Lagrangian and Eulerian description, J. Phys. Oceanogr., 24, 371-386, 1994.

Hansen, D. V. and Poulain, P.-M., Processing of WOCE/TOGA drifter data, J. Atmos. Oceanic Technol., 13, 900-909., 1996.

Lichtenberg, A. J. and M.A. Lieberman, M. A., Regular and Chaotic Dynamics, Springer-Verlag, 1992.

Orlic, M., Gacic, M., and La Violette, P. E., The currents and circulation of the Adriatic Sea, Oceanol. Acta, 15, 109-124, 1992.

Ottino, J. M., The kinematic of mixing: stretching, chaos and transport, Cambridge University, 1989.

Poulain, P.-M., Warn-Varnas, A., and Niiler, P. P., Near-surface circulation of the Nordic seas as measured by Lagrangian drifters, J. Geophys. Res., 101(C8), 18237-18258, 1996.

Poulain, P.-M., Drifter observations of surface circulation in the
Adriatic sea between December 1994 and March 1996, J. Mar. Sys., 20, 231-253, 1999.

Poulain, P.-M. and Zanasca, P., Drifter observation in the Adriatic sea (1994-1996). Data report, SACLANTCEN Memorandum, SM, SACLANT Undersea Research Centre, La Spezia, Italy, in press, 1998.

Samelson, R. M., Fluid exchange across a meandering Jet, J. Phys. Oceanogr., 22, 431, 1992.

Samelson, R. M., Chaotic transport by mesoscale motions, in R. J. Adler, P. Muller, B. L. Rozovskii (Eds.): Stochastic Modeling in Physical Oceanography, Birkhäuser, Boston, p. 423, 1996.

Taylor, G. I., Diffusion by continuous movements, Proc. Lond. Math Soc. (2) 20, 196-212, 1921.

Yang, H., Chaotic transport and mixing by ocean gyre circulation, in R. J. Adler, P. Muller, B. L. Rozovskii (Eds.): Stochastic Modeling in Physical Oceanography, Birkhäuser, Boston, p. 439, 1996.

Zore, M., On gradient currents in the Adriatic Sea. Acta Adriatic, 8 (6), 1-38, 1956. 